\title{
Analog of Fishtail Anomaly in Plastically Deformed Graphene
}

\author{
S. Sergeenkov and F.M. Araujo-Moreira \\ Grupo de Materiais e Dispositivos, Departamento de Física, Universidade Federal de S ao Carlos, 13565-905 S ao Carlos, SP, Brazil
}

By introducing a strain rate $\dot{\epsilon}$ generated pseudo-electric field $E_{x}^{d} \propto \hbar \dot{\epsilon}$, we discuss a magnetic response of a plastically deformed graphene. Our results demonstrate the appearance of dislocation induced paramagnetic moment in a zero applied magnetic field. More interestingly, it is shown that in the presence of the magnetoplastic effect, the resulting magnetization exhibits typical features of the so-called fishtail anomaly. The estimates of the model parameters suggest quite an optimistic possibility to experimentally realize the predicted phenomena in plastically deformed graphene.

PACS: 81.05.ue, 62.20.F-, 75.80.+q

1. Introduction. Many interesting and unusual phenomena due to significant modifications of the carbon based materials (including graphene) under mechanical deformations leading to generation of strong intrinsic pseudomagnetic fields have been recently discussed (see, e.g. [1, 2, 3, 4, 5] and further references therein). Of special interest for us are dislocations related properties in plastically deformed graphene and carbon nanotubes 6, 7, 8, 9.

In this Letter we consider theoretically some intriguing magnetic properties of graphene sheet under plastic deformations by directly incorporating a constant strain rate as a time dependent gauge potential into the Dirac model. The physics behind our findings and feasibility of their experimental verification are discussed.

2. Model. Recall [1, 2, 5] that in the absence of chirality (intervalley) mixing, the low-energy electronic properties of graphene near the Fermi surface can be reasonably described by a two-component wave function $|\Psi\rangle=\left(\Psi_{1}, \Psi_{2}\right)$ obeying a massless Dirac equation

$$
i \hbar \frac{\partial \mid \Psi>}{\partial t}=\mathcal{H} \mid \Psi>
$$

with an effective Hamiltonian

$$
\mathcal{H}=v_{F}\left(\sigma_{x} \pi_{x}+\sigma_{y} \pi_{y}\right)
$$

Here, $\pi_{a}=p_{a}+e A_{a}+e A_{a}^{d}$ with $p_{a}=-i \hbar \nabla_{a}$ being the momentum operator, $A_{a}$ the electromagnetic vector potential, and $A_{a}^{d}$ the deformation induced vector potential; $\sigma_{a}$ are the Pauli matrices, and $v_{F}$ is the Fermi velocity. In what follows, $a=\{x, y\}$. Let us consider a graphene sheet of length $L$ and width $W$ under the simultaneous influence of plastic deformation and perpendicular applied magnetic field $B_{z}$ (defined via the vector potential $\left.A_{y}=B_{z} x\right)$. As is well known [1, 2, 5], the homogeneous strain $\epsilon$ induced gauge potential, given by $e A_{x}^{d}=\hbar \epsilon / r$ (where $r=0.14 n m$ is carbon-carbon bond length), leads to appearance of intrinsic pseudomagnetic field $B_{z}^{d}=\hbar \epsilon / e L r$ inside deformed graphene lattice. By analogy with an applied electric field $E_{x}$ (defined via time-dependent vector potential $\left.A_{x}=E_{x} t\right)$, we introduce plastic deformation effects into the model through a constant plastic strain rate $\dot{\epsilon}$ dependent vector potential $A_{x}^{d}=E_{x}^{d} t$ resulting in appearance of intrinsic pseudo-electric field $E_{x}^{d}=\hbar \dot{\epsilon} / e r$.

3. Results and Discussion. Let us consider the magnetic response of the graphene sheet (with area $S=L W)$ on plastic deformation by analyzing its magnetization:

$$
M_{z}\left(B_{z}, \dot{\epsilon}\right) \equiv-\frac{1}{S}\left[\frac{\partial \mathcal{E}\left(B_{z}, \dot{\epsilon}\right)}{\partial B_{z}}\right]
$$

Here

$$
\mathcal{E}\left(B_{z}, \dot{\epsilon}\right)=\int_{0}^{\tau} \frac{d t}{\tau} \int_{0}^{L} \frac{d x}{L} \int_{0}^{W} \frac{d y}{W} \sum_{i=1}^{2}\left\langle\Psi_{i}|\mathcal{H}| \Psi_{i}\right\rangle
$$

is the total energy of the problem based on the previously obtained [5] solutions $\left|\Psi_{i}\right\rangle$ of time-dependent Eq.(1) ( $\tau$ is the characteristic time related to duration of plastic deformation, that is $\dot{\epsilon} \simeq \epsilon / \tau)$. First of all, the analysis of Eqs.(1)-(4) reveals that plastic deformation results in appearance of a non-zero magnetic moment $\mu_{z}=M_{z}(0, \dot{\epsilon}) S=\hbar v_{F} \tau \dot{\epsilon} / r$ in a zero applied magnetic field $\left(B_{z}=0\right)$. For typical experimental values of the applied strain rates [6, 9] $\dot{\epsilon} \simeq 10^{-4} s^{-1}$, we obtain $\mu_{z} \simeq 1 \mu_{B}$ for a reasonable estimate of the plastically induced paramagnetic moment in graphene [10] $\left(\mu_{B}\right.$ is the Bohr magneton). Fig. 1 shows the field dependence of the induced magnetization $\Delta M_{z}=M_{z}\left(B_{z}, \dot{\epsilon}\right)-M_{z}(0, \dot{\epsilon})$ for different values of the normalized strain rate $\dot{\epsilon}$ where $B_{0}=\Phi_{0} / S$ is a characteristic magnetic field ( $\Phi_{0}$ is the flux quantum). It is worth noting that it closely follows the observed [10] behavior of the point defects induced paramagnetic moment in graphene for different values 


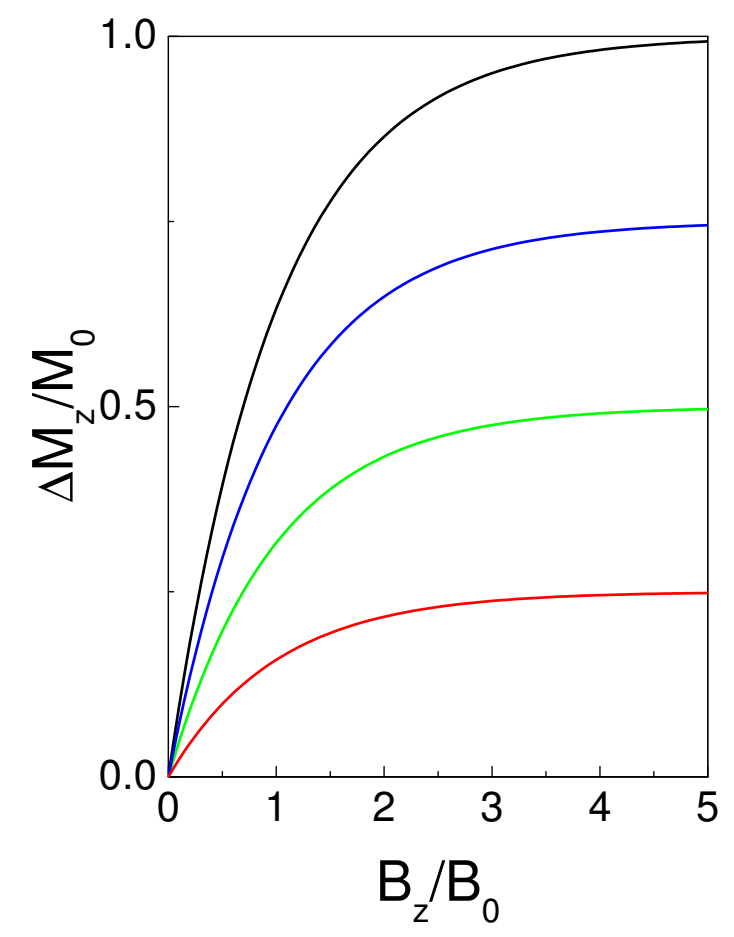

Fig 1. The magnetic field dependence of the normalized magnetization in plastically deformed graphene for different values of the normalized strain rate (from bottom to top): $\dot{\epsilon} / \dot{\epsilon}_{0}=0.25,0.5,0.75$, and 1.0. Here, $\dot{\epsilon}_{0}=10^{-4} s^{-1}$.

of density of vacancies $\rho_{v}$. This makes sense because plastic deformation is driven by motion of dislocations (with velocity $v_{d}$ ) leading to strain rate dependence on both $v_{d}$ and dislocation density $\rho_{d}$ as follows, $\dot{\epsilon}=b \rho_{d} v_{d}$. Here $b$ is the absolute value of the relevant Burgers vector. It is instructive to point out that the dislocation velocity $v_{d}$ in a sense plays a role of the Fermi velocity $v_{F}$ (which links applied electric and magnetic fields as $\left.E=v_{F} B\right)$ in relationship between strain rate induced pseudo-electric $E^{d}$ and strain induced pseudomagnetic $B^{d}$ fields. Indeed, with quite a good accuracy we can write $E^{d} \simeq v_{d} B^{d}$. At the same time, it is important to emphasize that (in addition to a definitely non-universal character of $v_{d}$ ) these two characteristic velocities describe phenomena on a completely different scale because while $v_{F} \simeq 10^{6} \mathrm{~m} / \mathrm{s}$, typical dislocation velocities rarely exceed $v_{d} \simeq 10^{-6} \mathrm{~m} / \mathrm{s}$. Let us turn now to another interesting phenomenon related to behavior of plastic deformation under applied magnetic field. Namely, as it was experimentally observed for many different types of materials (including semiconductors), upon application of magnetic field (of the order of $B=1 T$ ), dislocation velocity $v_{d}$ (and hence strain rate) becomes strongly field dependent. This phenomenon is called a magnetoplastic effect (MPE). It was discovered by Al'shits et al in 1987 [11. There are many different mechanisms which could be responsible for such a behavior [12. One of them (and probably most appropriate for graphene [10]) is based on interaction between uncompensated spin of dislocation's core and point paramagnetic impurities [13, 14 due to the difference in gyromagnetic factors $g$ (so-called $\Delta g$ mechanism) leading to appearance of resonance frequency $\omega_{r}=\Delta g \mu_{B} B / \hbar$ in applied magnetic field $B$. As a result, the interaction energy $U$ between dislocation and impurity becomes field dependent with $U(B)>U(0)$, which in turn leads to a significant increase of the thermally activated dislocation velocity described by the following expression [13, 14 .

$$
v_{d}(B)=v_{d}(0) \exp \left[\frac{\Delta U(B)}{k_{B} T}\right]
$$

where

$$
\Delta U(B)=U(B)-U(0)=U(0) f(B)
$$

with

$$
f(B)=\frac{B^{2}}{B^{2}+B_{p}^{2}}
$$

Here, $B_{p}=\hbar / \Delta g \mu_{B} \tau_{s}$ is the characteristic field for manifestation of MPE with $\tau_{s} \simeq \omega_{r}^{-1}\left(B=B_{p}\right)$ being the characteristic time. For typical values of $\Delta g \simeq 10^{-3}$ and $\tau_{s} \simeq 10^{-8} s$, we get $B_{p} \simeq 1 T$ for the estimate of the intrinsic magnetic field due to spin-mediated interaction between point and linear defects [11, 12, 13. So far, we have ignored the MPE in the magnetic response of graphene under plastical deformation. Let us see now what happens with magnetization in the presence of the above discussed MPE, that is assume that the strain rate becomes field dependent as follows, $\dot{\epsilon}\left(B_{z}\right)=b \rho_{d} v_{d}\left(B_{z}\right)$ with $v_{d}(B)$ given by Eq.(5). Notice that accounting for MPE virtually transforms our pseudo-electric field $E_{x}^{d}$ into a pseudo-magnetoelectric one $E_{x}^{d}\left(B_{z}\right)$. The obtained magnetic field dependence of the resulting magnetization is shown in Fig. 2 for $U(0)=0.01 k_{B} T, \dot{\epsilon}(0)=10^{-4} s^{-1}$ and for different values of the ratio $\gamma=B_{p} / B_{0}$ between two characteristic fields (notice that in Fig. 2 the applied field is normalized to $B_{p}$ instead of $B_{0}$ as in Fig. 1). We observe a remarkable fishtail like behavior of magnetization in plastically deformed graphene in the presence of MPE (a "diamagnetic" part of the curve $-M_{z} / M_{0}$ in Fig. 2 is added for better visual effects only). As it is clearly seen, the curve first reaches minimum at $B_{z} / B_{p} \simeq 1$, peaks around $B_{z} / B_{p} \simeq 2$ and then gradually diminishes at higher applied fields. Recall that such a behavior has been observed before in superconductors and 


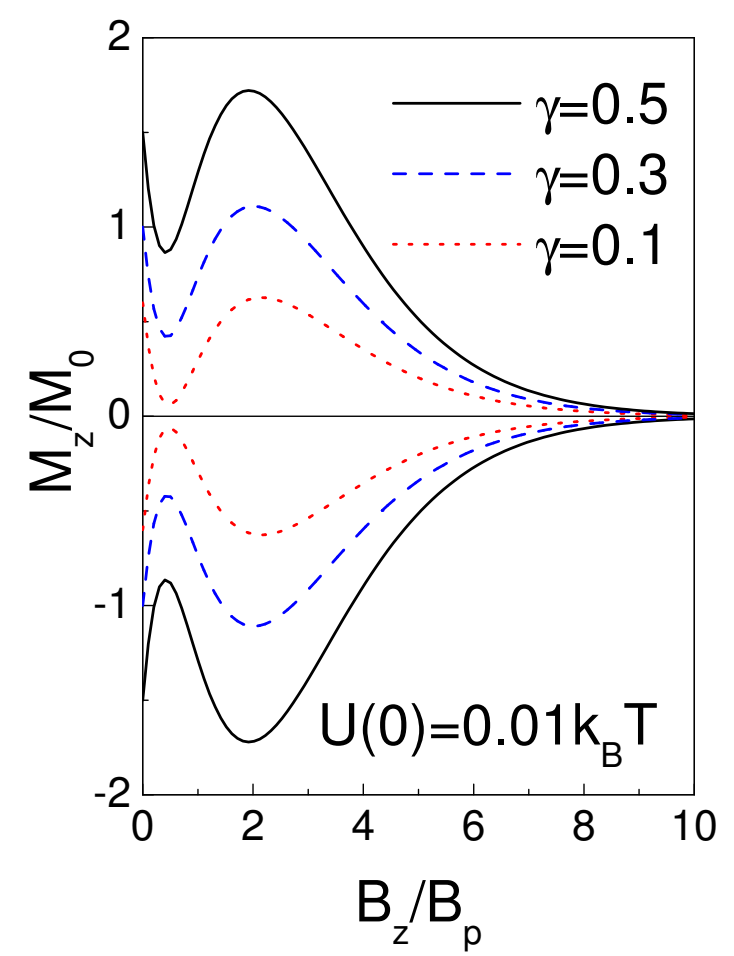

Fig 2. The magnetic field dependence of the normalized magnetization in the presence of magnetoplastic effect for $U(0)=0.01 k_{B} T, \dot{\epsilon}(0)=10^{-4} s^{-1}$ and three values of $\gamma=B_{p} / B_{0}$. A "diamagnetic" part of the curve $-M_{z} / M_{0}$ is added for better visual effects only.

attributed to a perfect match between the sizes of the vortex core and the pinning center. While for Abrikosov vortices the best pins are point defects (vacancies) [15], the so-called Josephson vortices (fluxons) require linear (or even planar) defects for their effective pinning [16]. By analogy, we can assume that the discussed here MPE induced fishtail anomaly in graphene structure probably has something to do with a perfect match (energy minimization) between a paramagnetic impurity and magnetic field modified dislocation, which serves as a spinsensitive pinning center for this impurity [7, 8, 10, For typical values of the width $W=40 \mathrm{~nm}$ and aspect ratio $L / W=10$ we obtain $B_{0}=\Phi_{0} / W L \simeq 10 T$ for the estimate of the characteristic field in graphene (shown in Fig. 1) which should be compared with the earlier estimated value of the MPE mediated intrinsic magnetic field $B_{p} \simeq 1 T$. According to Fig. 2 the fishtail like behavior is expected to manifest itself already for $\gamma=B_{p} / B_{0} \geq 0.1$ which makes the experimental observation of the predicted here phenomena quite feasible.

In summary, by incorporating strain rate as a gauge potential (for pseudo-electric fields) into the model describing low-energy electron properties in graphene, we have calculated magnetic response of a plastically deformed graphene. According to our findings, under such deformations graphene acquires dislocation induced paramagnetic moment in a zero applied magnetic field. Besides, in the presence of the so-called magnetoplastic effect (when the strain rate becomes strongly dependent on the applied magnetic field), the resulting magnetization was found to exhibit typical features of the so-called fishtail anomaly, attributed to spindependent interaction between dislocations and paramagnetic impurities in plastically deformed graphene.

This work has been financially supported by the Brazilian agencies CAPES, CNPq, and FAPESP.

1. M.A.H. Vozmediano, M.I. Katsnelson, and F. Guinea, Phys. Rep. 496, 109 (2010).

2. Ken-ichi Sasaki, Riichiro Saito, M.S. Dresselhaus, Katsunori Wakabayashi, and Toshiaki Enok, New Journal of Phys. 12, 103015 (2010).

3. N. Levy, S.A. Burke, K.L. Meaker, M. Panlasigui, A. Zettl, F. Guinea, A. H. Castro Neto, and M. F. Crommie, Science 329, 544 (2010).

4. L.T. Singh, S. Bhattacharyya, Abhishek K. Singh, and K. K. Nanda, Phys. Rev. Lett. 110, 095504 (2013).

5. S. Sergeenkov and F.M. Araujo-Moreira, Solid State Commun. 158, 58 (2013).

6. Yoshikazu Nakayama, Jap. J. Appl. Phys. 46, 5005 (2007).

7. Oleg V. Yazyev and Steven G. Louie, Phys. Rev. B 81, 195420 (2010).

8. O. V. Yazyev, Rep. Prog. Phys. 73, 056501 (2010).

9. Jamie H. Warner, Elena Roxana Margine, Masaki Mukai, Alexander W. Robertson, Feliciano Giustino, and Angus I. Kirkland, Science 337, 209 (2012).

10. R. R. Nair, M. Sepioni, I-Ling Tsai, O. Lehtinen, J. Keinonen, A. V. Krasheninnikov, T. Thomson, A.K. Geim, and I.V. Grigorieva, Nature Physics 8, 199 (2012).

11. V. I. Al'shits, E. V. Darinskaya, T. M. Perekalina, and A. A. Urusovskii, Sov. Phys. Solid State 29, 265 (1987).

12. R.B. Morgunov, Physics Uspekhi 47, 125 (2004).

13. M.I. Molotskii, Sov. Phys. Solid State 33, 1760 (1991).

14. V. I. Al'shits and E. V. Darinskaya, JETP Lett. 70, 761 (1999).

15. D. Daeumling, J. M. Seuntjens, and D.C. Larbalestier, Nature 346, 332 (1990).

16. S. Sergeenkov, L.Jr. Cichetto, V.A.G. Rivera, C. Stari, E. Marega, C.A. Cardoso and F.M. Araujo-Moreira, JETP Lett. 91, 30 (2010). 